\title{
Association between early cerebral oxygenation and neurodevelopmental impairment or death in premature infants
}

\author{
Anup C. Katheria $\mathbb{D}^{1} \cdot$ Jacob Stout $^{1} \cdot$ Ana L. Morales ${ }^{1} \cdot$ Debra Poeltler $^{1} \cdot$ Wade D. Rich $^{1} \cdot$ Jane Steen $^{1} \cdot$ \\ Shauna Nuzzo ${ }^{1} \cdot$ Neil Finer $^{1}$
}

Received: 6 July 2020 / Revised: 15 October 2020 / Accepted: 19 January 2021 / Published online: 15 February 2021

(c) The Author(s), under exclusive licence to Springer Nature America, Inc. 2021

\begin{abstract}
Objective To assess the relationship between cerebral oxygenation in the first $72 \mathrm{~h}$ of life and neurodevelopmental impairment (NDI) at 2 years corrected age in former premature infants.

Study design Prospective observational cohort study of 127 infants $<32$ weeks GA at birth with cerebral oxygenation monitoring using NIRS in the first $72 \mathrm{~h}$ of life.

Results Using a threshold cutoff for cerebral hypoxia, infants with NDI or death had increased duration of hypoxia (4 vs $2.3 \%, p=0.001$ ), which was more pronounced in the $23-27$ week subgroup ( 7.6 vs $3.2 \%, p<0.001$ ). Individual generalized estimating equations to adjust for repeated measures were modeled in this subgroup for the physiologic parameters including $\mathrm{StO}_{2} . \mathrm{StO}_{2}<67 \%$ was a predictor for death or NDI (OR 2.75, 95\% CI 1.006, 7.5132, $p=0.049$ ).

Conclusion An increased duration of cerebral hypoxia is associated with NDI or death in infants born $<32$ weeks GA.
\end{abstract}

\section{Introduction}

Underdeveloped cerebral circulatory systems of preterm infants have limited cerebral autoregulation that increase their risk of severe complications such as intraventricular hemorrhage (IVH) or death. Current standard monitoring in the delivery room and NICU include heart rate (HR) and peripheral arterial oxygen saturation $\left(\mathrm{SpO}_{2}\right)$, but neither provide a direct measure of cerebral oxygenation. Non-invasive monitoring of cerebral tissue oxygen saturation $\left(\mathrm{StO}_{2}\right)$ using near-infrared spectroscopy (NIRS) during resuscitation and neonatal transition may present a way to identify patients at the highest risk of impairment in the early days of life.

A previous single center European study has identified an association between cerebral oxygenation and developmental outcomes but this result has not been replicated [1]. A few small studies have explored NIRS value ranges for the prediction of neurodevelopmental outcomes in the

Anup C. Katheria

anup.katheria@sharp.com

1 Neonatal Research Institute, Sharp Mary Birch Hospital for Women \& Newborns, San Diego, CA, USA context of neonatal morbidities like hypoxic ischemic encephalopathy (HIE), late-onset sepsis, and congenital heart disease. However, these studies were unable to show correlation between NIRS and developmental outcomes [2-4]. Additionally, 83 preterm infants in the SafeBoosC II trial followed to 2 years of age showed no developmental benefit on the Bayley 2 or 3 examination when cerebral oximetry was included in the treatment of extremely preterm infants [5].

Our prospective observational cohort study of neuromonitoring preterm infants during the first $72 \mathrm{~h}$ of life (The Neu-Prem Trial) demonstrated that NIRS monitoring during resuscitation held potential for predicting IVH or death [6]. The study found that differences in $\mathrm{StO}_{2}$ within the first $24 \mathrm{~h}$ of life were predictive of the development of any grade IVH and death. Given the relationship between cerebral oxygenation and IVH or death, we hypothesized that cerebral oxygenation in the first $72 \mathrm{~h}$ of life could predict neurodevelopmental impairment (NDI) at 2 years of age. A better understanding of the relationship between neonatal cerebral oxygenation and neurodevelopmental outcomes may provide guidelines for improved monitoring of preterm newborns, potentially improving neurodevelopmental outcomes. Different devices have varied thresholds for cerebral hypoxia. The suggested threshold for the Fore-Site Elite monitor used in our study calibrated using a phantom model 
is $67 \%$ when using a neonatal probe [7]. We hypothesized that there were differences in duration of cerebral hypoxia in newborns that developed NDI or death when compared to those that did not show any signs of impairment at 2 years of age.

\section{Methods}

The original prospective cohort trial was conducted from October 2015 through December 2016 and has been previously published [6]. Informed consent was obtained for the study and subsequent neurodevelopmental follow-up. As part of the original trial a NIRS sensor and electroencephalography leads were placed on 127 newborns $<32$ weeks of gestational age at birth. Data recorded in the delivery room included ten minutes of continuous NIRS and aEEG along with heart rate, peripheral arterial oxygen saturation $\left(\mathrm{SpO}_{2}\right)$, fraction of inspired oxygen, and mean airway pressure measurements. Data were recorded in the neonatal intensive care unit for $72 \mathrm{~h}$ and included, NIRS, aEEG, and vital signs (heart rate, blood pressure, and $\mathrm{SpO}_{2}$ ). Within the first $12 \mathrm{~h}$ of life and again at $72 \mathrm{~h}$ of life an ultrasound scan of the head was performed.

Survivors of the original cohort were followed out to 22-26 months corrected age in our high-risk infant follow up clinic to assess for our primary outcome of NDI or death. Due to this being a convenience sample we were unable to calculate a sample size. Criteria for inclusion in this analysis matched those used for inclusion in the original trial- included infants born $<32$ weeks GA and did not have congenital anomalies or moderate-to-severe head bruising at birth.

The 22- to 26-month corrected age follow-up visit included a health history, physical examination, and cognitive, language, and motor assessment using the Bayley Scales of Infant and Toddler Development, third edition (Bayley-III), a standardized neurologic examination, and measurements (weight, height, and head circumference). The Gross Motor Function Classification System (GMFCS) was used to evaluate functional motor outcomes. Neurodevelopmental assessment was carried out by examiners who were trained in administration of the Bayley-III, had excellent inter-rater reliability (0.90), and masked to the NIRS values. The Bayley-III includes cognitive, language (receptive and expressive subscales), and motor (fine and gross motor subscales) composite scores with a mean and SD of $100 \pm 15$. The GMFCS, as modified by the National Institute of Child Health and Human Development Neonatal Research Network [8], is a validated system that can be used for children between 22 and 26 months CA to describe the severity of motor dysfunction. GMFCS levels range from 0 to 5 with a level of 1 indicating mild impairment and a level of 5 indicating the most severe motor impairment. Parental interviews conducted at 2 years of age were used to obtained data regarding visual (e.g., eye surgery, strabismus, myopia) or hearing impairments, pulmonary morbidity including the need for oral or inhaled steroids, oxygen, and bronchodilators, and rehospitalizations.

Moderate to severe NDI defined as having $\geq 1$ of the following: a Cognitive composite score of $<70$, GMFCS of $\geq 2$, blindness (vision of $<20 / 200$ ), or hearing impairment interfering with the ability to communicate with amplification. Mild NDI was defined as having a Cognitive score of $<85$, GMFCS score of 1 or more, at least unilateral blindness (vision of 20/200 in only 1 eye), hearing impairment, or need for hearing aids.

\section{Statistical analyses}

The sample consisted of infants with $\mathrm{StO}_{2}$ data for the first $72 \mathrm{~h}$ and two year follow up data available as well as those who died during hospitalization. Data were averaged hourly for each subject and generalized estimating equations were then used to control for repeated measures. We used an hourly $\mathrm{StO}_{2}$ average of 67 as the threshold cutoff. We performed a secondary analysis of delivery room $\mathrm{StO}_{2}$ (first $10 \mathrm{~min}$ of life) and each of the individual outcomes of cognitive, language and motor impairment or death. All data were analyzed using the hypoxic threshold of 67 identified during the original trial [6], and calibration data from Kleiser et al. [7].

Analysis was conducted using SPSS (IBM Corp. Released 2016. SPSS Statistics for Windows, Version 24, Armonk, New York, USA) and Epi-Info ${ }^{\mathrm{TM}} 7.0$ (CDC, Atlanta, GA, USA). Before analysis, data was first verified for accurate data entry, formats, coding, and missing observations. The largest and smallest values for each variable were reviewed for accuracy and plausibility. Each variable was examined for variability and frequency distribution, skewness, and kurtosis. Subsequent data analyses were conducted using descriptive, univariable, and adjusted analyses. Repeated measures analyses were conducted related to specific study aims. All significance tests were 2sided with critical alpha level of 0.05. Comparison of demographic and subject characteristics were made using Chi-Square, Fisher's exact test, or logistic regression for dichotomous and categorical variables. Student's $t$ tests for comparison of means were used for continuous variables, and Mann-Whitney U or Wilcoxon rank-sum test was used as a nonparametric test for ordered categorical variables or for continuous variables failing to meet normal distribution requirements.

Univariable and adjusted analyses between the dependent variable and each independent variable were examined to explore the crude associations between variables using 


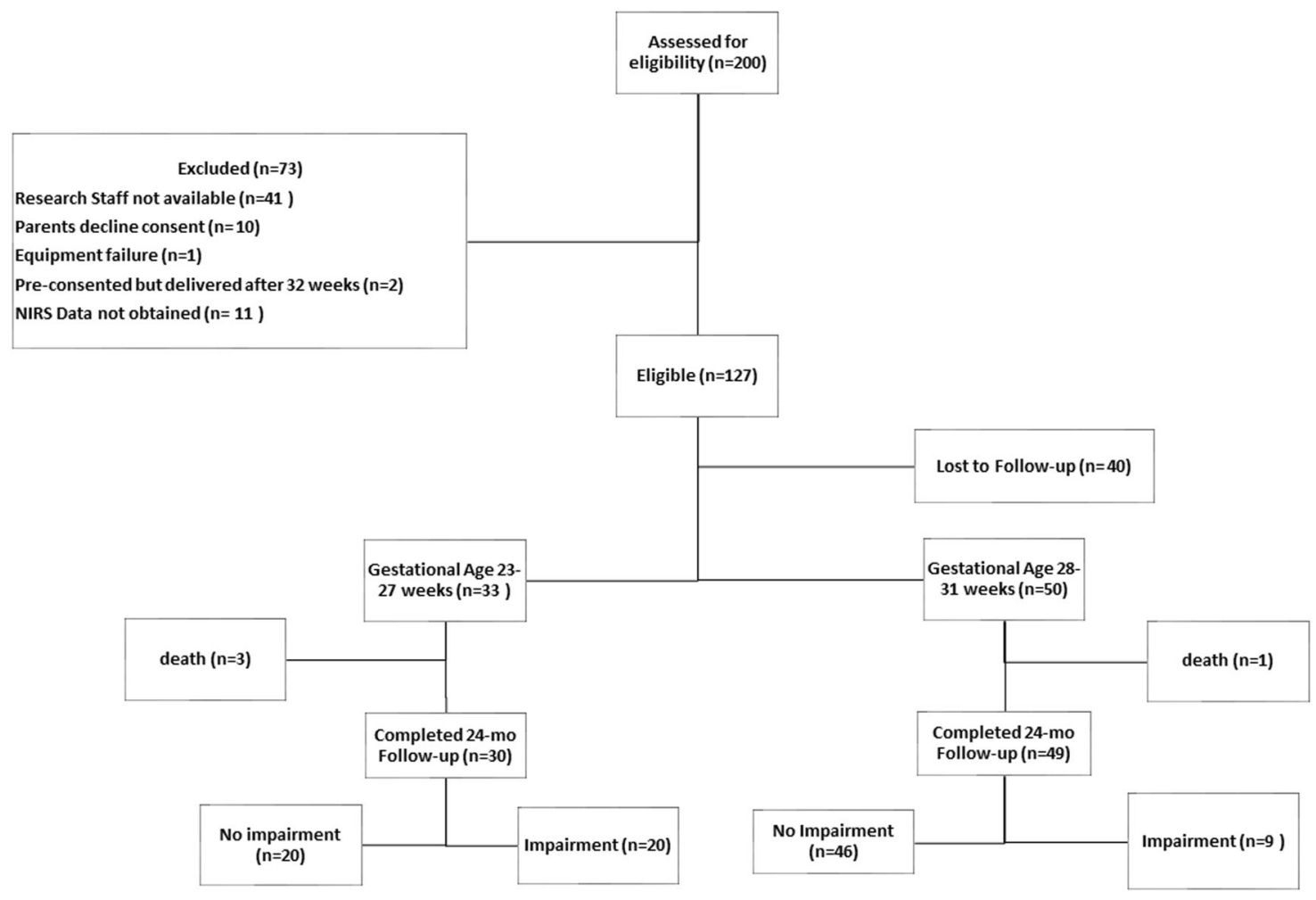

Fig. 1 Consort diagram. Progression of Subjects through screening, randomization and followup.

Chi-Square, Fisher's exact test, ANOVA, Mann-Whitney U or Wilcoxon rank-sum test, linear regression, or logistic regression. Repeated measures analyses were performed via generalized estimating equation modeling (GEE).

\section{Results}

From the original cohort of 127 infants, 79 children were able to return for their neurodevelopmental follow-up exam at 2 years corrected age between June 2017 and February 2019 (range 20-26 months corrected). There were 4 infants who died prior to follow-up examination and were included in the composite outcome of any disability or death at 2-year follow up. Of the total sample of 83 infants, 33 were born between 23 and 27 weeks GA and 50 were born between 28 and 31 weeks gestational age at birth (see Fig. 1).

For the primary outcome, using the $\mathrm{StO}_{2}$ threshold of $67 \%$, infants with NDI or death had increased duration of cerebral hypoxia ( 4 vs $2.3 \%, p<0.001)$. For the individual components of the neurodevelopmental exam, there was an increased duration of cerebral hypoxia in subjects with language impairment ( 3.4 vs $2.4 \%, p=0.042$ ), death 4.3 vs $3.7 \% p=0.049$, but not cognitive or motor impairment, or death. The overall cohort neonatal demographics are shown in Table 1.
Table 1 Overall cohort neonatal demographics.

\begin{tabular}{lc}
\hline Sample $n=83$ & \\
\hline Gestational age mean (sd) & $28.54(2.58)$ \\
Gestational age 23-27 weeks (\%) & $33(40)$ \\
Male (\%) & $44(53)$ \\
Any IVH (\%) & $15(18)$ \\
Severe IVH (\%) & $3(4)$ \\
Death (\%) & $4(5)$ \\
Completed follow-up: $n=79$ & \\
Moderate to severe developmental impairment $n(\%)$ & $3(4)$ \\
Mild cognitive impairment $(\%)$ & $5(6)$ \\
Mild language impairment $(\%)$ & $24(30)$ \\
Mild motor impairment $(\%)$ & $9(11)$ \\
Any impairment or death $(\%)$ & $29(35)$ \\
\hline
\end{tabular}

A subgroup analysis of infants 23-27 weeks GA with NDI or death demonstrated a greater duration and difference of cerebral hypoxia (7.6 vs 3.2 ( $p<0.001$, OR $2.470,95 \%$ CI 1.644, 3.712) compared to those without NDI or death. There was no statistically significant difference between the impaired and non-impaired groups in neonatal or maternal characteristics (Table 2).

There were no differences in other vital signs in the first $72 \mathrm{~h}$ of life (Fig. 2) in the 23-27 week cohort. Individual generalized estimating equations to adjust for repeated 
measures were modeled in this subgroup for the physiologic parameters $\mathrm{StO}_{2}<67$, heart rate, $\mathrm{SpO}_{2}, \mathrm{IVH}$ and mean arterial pressure (Table 3 ) over the first $72 \mathrm{~h}$ of life. $\mathrm{StO}_{2}<67$ was the

Table 2 Maternal and neonatal characteristics.

\begin{tabular}{lcr}
\hline $\begin{array}{l}\text { Gestational age 23-27 weeks } \\
n=33\end{array}$ & $\begin{array}{l}\text { Impairment } \\
\text { or death } \\
\text { Yes } n=13\end{array}$ & No $n=20$ \\
\hline Maternal age mean (sd) & $29(7)$ & $30(6)$ \\
Mode of delivery Cesarean $n(\%)$ & $10(77)$ & $20(100)$ \\
Preterm labor $n(\%)$ & $11(85)$ & $16(80)$ \\
Chorioamnionitis $n(\%)$ & $7(54)$ & $12(60)$ \\
Preeclampsia $n(\%)$ & $2(15)$ & $3(15)$ \\
Maternal diabetes $n(\%)$ & $2(15)$ & $4(20)$ \\
Multiple delivery $n(\%)$ & $4(31)$ & $9(45)$ \\
At least 1 dose antenatal steroids & $10(77)$ & $20(100)$ \\
$n$ (\%) & $25(1.4)$ & $25(1.5)$ \\
Gestational age & $817(248)$ & $758(238)$ \\
Birthweight mean (sd) & $26.08(29.2)$ & $17.75(14.9)$ \\
Days on ventilator mean (sd) & $104(25)$ \\
Length of stay NICU mean (sd) & $104(72)$ & $11(55)$ \\
Gender male $n$ (\%) & $6(46)$ & $5(25)$ \\
Any IVH $n$ (\%) & $4(31)$ & $5(3,7)$ \\
Apgar 1 min, median (IQR) & $5(3,6)$ & $7(6,8)$ \\
Apgar 5 min, median (IQR) & $8(6,8)$ & \\
\hline
\end{tabular}

only predictor for death or neurodevelopmental impairment (OR 2.75, 95\% CI 1.006, 7.5132, $p=0.049$ ).

A secondary analysis of delivery room $\mathrm{StO}_{2}$ in the first $10 \mathrm{~min}$ of life for each of the individual outcomes and/or the combined outcomes of cognitive, language and motor impairment or death did not demonstrate any association (data not shown).

\section{Discussion}

Several cohort studies in Europe that used the INVOS monitor have found an association between low cerebral oxygenation in the first few hours/days of birth and poor long-term outcomes. Some have used a pediatric sensor or

Table 3 Individual generalized estimating equations to predict any impairment or death, 23-27 weeks GA, 72 hours, adjusting for any IVH.

\begin{tabular}{lrllll}
\hline & \multicolumn{1}{l}{$B$} & SE & $p$ & OR & $95 \%$ CI \\
\hline Any IVH & -0.021 & 0.8534 & 0.980 & 0.979 & $0.184,5.215$ \\
$\mathrm{St}_{2}<67$ & 1.011 & 0.5128 & 0.049 & 2.75 & $1.006,7.5132$ \\
$\mathrm{HR}$ & 0.025 & 0.0255 & 0.32 & 1.026 & $0.976,1.078$ \\
$\mathrm{Sp}_{2}$ & -0.006 & 0.666 & 0.928 & 0.994 & $0.872,1.132$ \\
$\mathrm{MAP}$ & 0.036 & 0.0495 & 0.455 & 1.037 & $0.941,1.142$ \\
\hline
\end{tabular}

A
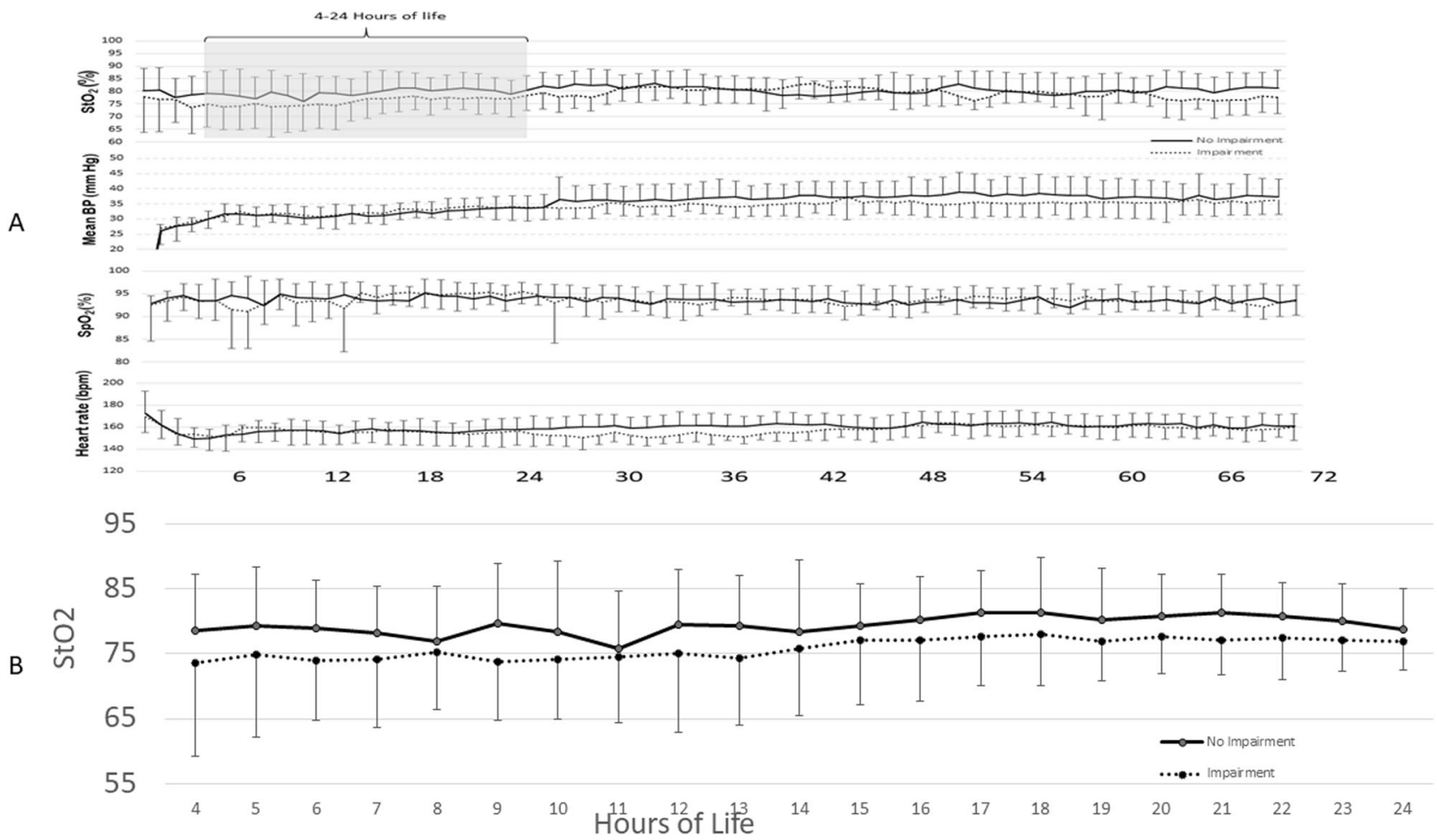

Fig. 2 Physiological parameters presented over time in the 23-27 week cohort. Cerebral oximetry $\left(\mathrm{StO}_{2}\right)$, mean blood pressure (BP), peripheral oxygen saturation $\left(\mathrm{SpO}_{2}\right)$ and heart rate (beats per minute) over $72 \mathrm{~h}$ (Panel A) and from 4 to $24 \mathrm{~h}$ of age (Panel B). 
an adult sensor with different cutoff values $(65 \%$ for the neonatal sensors and $55 \%$ for the adult sensor). Three cohort studies to date have demonstrated impairment with abnormal cerebral oxygenation. Verhagen et al. followed 67 infants (mean gestational age 30 weeks) with cerebral oximetry daily (days 1, 2, 3, 4, 5, 8 and 15) and found poorer cognitive outcome with lowest (25-50) and highest quartile (75-100) $\mathrm{StO}_{2}$ [9]. In addition, the lowest quartile (25-50) was also associated with poorer fine motor outcome. Alderliesten et al. compared a cohort of 66 preterm infants $<32$ weeks treated for hypotension to a set of matched controls that were not treated [10]. While the receipt of blood pressure treatment did not affect neurodevelopmental outcomes, 26 infants with a $\mathrm{StO}_{2}$ of $<50 \%$ for $>10 \%$ of the time had a lower neurodevelopmental scores at 18 months (median 99 vs $104, P=0.02)$. Recently the same group published a larger cohort $(N=221$ for 24-27 week CGA infants seen at 2 years) demonstrating that low $\mathrm{StO}_{2}$ was associated with an unfavorable cognitive outcome [1]. They recommended the use of a threshold of $\mathrm{StO}_{2}<55 \%$ for future clinical studies when using the adult near-infrared sensors $\left(\mathrm{StO}_{2}<65 \%\right.$ for neonatal sensor). All three studies used the INVOS devices.

Different near-infrared spectroscopy monitors use different technologies and algorithms to obtain results, and consequently show readings which are not the same for an identical physiologic circumstance [11]. Investigators have attempted to use both in-vivo [12] and in-vitro [7, 13] methods to validate these results. Based on the use of a single device, the SafeBoosc trial used a normal range of clinical values to be 55-85\% using a single device [14]. When calibrated to the INVOS device using a phantom, the suggested threshold for the Fore-Site Elite monitor used in this study is 67 when using a neonatal probe [7]. We chose to analyze this data by using a cutoff value of 67 as this best represented a clinical comparison useful to clinicians.

Limitations to this study include a small sample size due to a number of infants being lost to follow-up. The 2-year cognitive assessment was not the primary outcome of the original trial and therefore extensive retention measures were not in place at the onset of the trial. Only $64 \%$ of infants eligible for neurodevelopmental follow-up completed a cognitive assessment at 2 years of age. Other limitations include an inability to correct for multiplicity due our small sample size. Furthermore, selection bias may be present in the analyzed group because infants that did not complete cognitive assessment may have been assessed by parents as not in need of support services. There is a concern for missed cognitive assessment for infants of older gestations (>30 weeks) who are more likely to be assessed by parents as normally developing.

It remains unclear whether we can improve neurodevelopmental outcomes of preterm infants by intervening on a low $\mathrm{StO}_{2}$. Current efforts to intervene on low $\mathrm{StO}_{2}$ include the SafeBoosc III trial, which plans to determine whether intervening on a low NIRS can reduce risk of death or severe brain injury [15]. The study was designed to use saturation targets based on values from the INVOS device but will allow for the use of the FORESITE elite at the cutoff level of 67 for cerebral hypoxia. Ideally, the SafeBoosc III trial will have enough power to determine whether intervening on a low NIRS using each device can successfully reduce both the short-term morbidities (death and severe brain injury) as well as longterm neurodevelopmental impairment. Our follow-up of the original Neuprem cohort is the first to demonstrate an association between the increased time spent below a threshold of 67 using the FORESITE device and an abnormal neurodevelopmental outcome or death in preterm infants.

Funding All phases of this study were supported by the Gerber Foundation and the Norris Foundation. Near InfraRed Spectroscopy devices (FORESITE) were provided to all sites by Casmed Medical Systems, Inc. Neither funding source had any involvement in the study design, data collection, or data analysis. This data has not been presented anywhere.

Author contributions ACK conceptualized and designed the study, drafted the initial manuscript, designed the data collection instruments, and coordinated and supervised data collection, and approved the final manuscript as submitted. DP and NF carried out the initial analyses, reviewed and revised the manuscript, and approved the final manuscript as submitted. WDR, JS, AM, JS and SN collected the data, carried out the initial analyses, reviewed and revised the manuscript, and approved the final manuscript as submitted. All authors approved the final manuscript as submitted and agree to be accountable for all aspects of the work.

\section{Compliance with ethical standards}

Conflict of interest The authors declares no competing interests.

Publisher's note Springer Nature remains neutral with regard to jurisdictional claims in published maps and institutional affiliations.

\section{References}

1. Alderliesten $T$, van Bel $F$, van der Aa NE, Steendijk $P$, van Haastert IC, de Vries LS, et al. Low cerebral oxygenation in preterm infants is associated with adverse neurodevelopmental outcome. J Pediatrics. 2019;207:109-116.e102.

2. Burton VJ, Gerner G, Cristofalo E, Chung SE, Jennings JM, Parkinson C, et al. A pilot cohort study of cerebral autoregulation and 2-year neurodevelopmental outcomes in neonates with hypoxicischemic encephalopathy who received therapeutic hypothermia. BMC Neurol. 2015;15:209.

3. Zonnenberg IA, Dijk JV, Dungen F, Vermeulen RJ, Weissenbruch MMV. The prognostic value of NIRS in preterm infants with (suspected) late-onset sepsis in relation to long term outcome: a pilot study. PloS one. 2019;14:e0220044. 
4. Simons J, Sood ED, Derby CD, Pizarro C. Predictive value of near-infrared spectroscopy on neurodevelopmental outcome after surgery for congenital heart disease in infancy. $\mathrm{J}$ Thorac Cardiovasc Surg. 2012;143:118-25.

5. Plomgaard AM, Alderliesten $\mathrm{T}$, van Bel F, Benders $\mathrm{M}$, Claris O, Cordeiro M, et al. No neurodevelopmental benefit of cerebral oximetry in the first randomised trial (SafeBoosC II) in preterm infants during the first days of life. Acta Paediatr. 2019;108:275-81.

6. Katheria AC, Harbert MJ, Nagaraj SB, Arnell K, Poeltler DM, Brown MK, et al. The Neu-Prem Trial: neuromonitoring of brains of infants born preterm during resuscitation-a prospective observational cohort study. J Pediatrics. 2018;198:209-213.e203.

7. Kleiser S, Ostojic D, Andresen B, Nasseri N, Isler H, Scholkmann $\mathrm{F}$, et al. Comparison of tissue oximeters on a liquid phantom with adjustable optical properties: an extension. Biomed Opt Express. 2018;9:86-101.

8. Palisano R, Rosenbaum P, Walter S, Russell D, Wood E, Galuppi B. Development and reliability of a system to classify gross motor function in children with cerebral palsy. Dev Med Child Neurol. 1997;39:214-23.

9. Verhagen EA, KNJA VanBraeckel, van der VeereCN, Groen H, Dijk PH, Hulzebos CV, et al. Cerebral oxygenation is associated with neurodevelopmental outcome of preterm children at age 2 to 3 years. Dev Med Child Neurol. 2015;57:449-55.
10. Alderliesten T, Lemmers PM, van Haastert IC, de Vries LS, Bonestroo HJ, Baerts W, et al. Hypotension in preterm neonates: low blood pressure alone does not affect neurodevelopmental outcome. J Pediatr. 2014;164:986-91.

11. Bickler PE, Feiner JR, Rollins MD. Factors affecting the performance of 5 cerebral oximeters during hypoxia in healthy volunteers. Anesth Analg. 2013;117:813-23.

12. Benni PB, MacLeod D, Ikeda K, Lin HM. A validation method for near-infrared spectroscopy based tissue oximeters for cerebral and somatic tissue oxygen saturation measurements. J Clin Monit Comput. 2018;32:269-84.

13. Hyttel-Sorensen S, Kleiser S, Wolf M, Greisen G. Calibration of a prototype NIRS oximeter against two commercial devices on a blood-lipid phantom. Biomed Opt Express. 2013;4:1662-72.

14. Hyttel-Sorensen S, Austin T, van Bel F, Benders M, Claris O, Dempsey E, et al. A phase II randomized clinical trial on cerebral near-infrared spectroscopy plus a treatment guideline versus treatment as usual for extremely preterm infants during the first three days of life (SafeBoosC): study protocol for a randomized controlled trial. Trials. 2013;14:120.

15. Hansen ML, Pellicer A, Gluud C, Dempsey E, Mintzer J, HyttelSørensen S, et al. Cerebral near-infrared spectroscopy monitoring versus treatment as usual for extremely preterm infants: a protocol for the SafeBoosC randomised clinical phase III trial. Trials. 2019; 20:811. 